\title{
Versatile Detector of Pseudo Periodic Patterns
}

\author{
Augusto Santini ${ }^{1}$, Emiliano Diez $^{2}$, Mariano Llamedo ${ }^{1}$ \\ ${ }^{1}$ GIBIO, National Technological University, Buenos Aires, Argentina \\ ${ }^{2}$ Institute of Physiology, Medical School, National University of Cuyo, Mendoza, Argentina
}

\begin{abstract}
This study aimed to develop a detector with few physiologically-meaningful parameters, that could be capable of detecting pseudo periodic patterns. The algorithm is based in a signal detection based on a matched filter, and a threshold calculation based on robust stadistics. The evaluation of the detector was performed under a corpus consisting in two sets. One set of human ECGs, and one set of rodent pseudo ECGs. The evaluation was performed with respect to the gold standard annotations, and was calculated in terms of sensitivity $(S)$ and positive predictive value $(P)$. For the human ECG set of recordings, the detector had $100 \mathrm{~S}$ and $99.9 \mathrm{P}$, while for the rodent pseudo ECG set of recordings the results where 97.1 S and 79.1 P. Both sets results are median values. The results obtained under the rodent set where compared to those obtained with another two detectors, where our detector obtained the best results. The algorithm achieved promising results, in a broad set of ECG recordings of very different nature, with the additional capability of further adaptation provided by expert assistance.
\end{abstract}

\section{Introduction}

The automatic analysis of pseudo periodic signals, such as those of cardiovascular origin, often relies on the detection of a synchronization pattern in order to perform a reliable estimation through averaging. In certain applications such as fetal ECG analysis or transmembrane action potential measurement, the automatic synchronization task can be a challenging problem by itself. The detections of heartbeats in the ECG can also be a challenging task, specially in those long-term and/or noisy recordings, with broad and sudden changes in rhythm and morphology. The success commonly relies on the a priori knowledge about the timing and morphologic description about the pattern to detect, therefore limiting the generality.

In the past two decades several cardiovascular pattern detectors were developed, e.g. ECG heartbeat detectors, blood pressure pulse detectors, etc. Some of them adapted for noninvasive signals such as the ECG or the plethysmo- graphic signal, but also for other invasive signals, such as the electrogram and blood pressure recordings, among others. Several of the developed detectors are freely available on internet [1], and were described in the literature [2,3]. All the reviewed algorithms are commonly adapted to an specific type of pattern/signal, making difficult or impossible the possibility of changing the target pattern and/or signal. As a consequence, these algorithms have a limited adaptation capacity to real life applications, where noise interference and large morphological changes in the signal/patterns often occur.

The objective of this work is to develop and analyze an algorithm to detect impulsive and pseudo periodic patterns in cardiovascular signals given a small set of timing and morphological constraints. In addition, it must be capable of both automatic and assisted operation, i.e. the algorithm must provide a simple interface for an eventual expert.

\section{Material and methods}

The performance of the algorithm was evaluated in a data corpus consisting in two evaluation sets. The first evaluation set consists in 178 human ECG recordings from 12 publicly available databases [1]. Details of the human set are shown in Table 1. The second evaluation set consists in 17 pseudo ECG recordings, performed in explanted rodent hearts put through hipokalemia [4]. The recordings length were between 20 and 50 minutes, accounting for a total time of 10 hours aproximately. For all ECG recordings, only lead II or the first available was selected for experimentation.

The algorithm proposed in this work is mainly based on the analysis of a detection signal calculated as

$$
a(\theta)=\sum_{i=\theta}^{\theta+L-1} s(i) \cdot p(\theta-i), \quad \theta=0,1 \cdots, N
$$

where $p(n)$ (p hereafter) is a pattern of length $N$, and $\mathbf{s}$ the signal under analysis, of $N$ samples length. This method is also known as the matched filter [2], where $\mathbf{p}$ is the time reversed version of the pattern to detect. The matched filter is the maximum likelihood delay estimator $(\theta)$ of a pattern 
Table 1. Human evaluation set composition.

\begin{tabular}{ccccc} 
Group & Name & Length & \#Rec & Leads \\
\hline \multirow{2}{*}{ Sinus } & nsrdb & 1 day & 17 & 2 \\
& fantasiadb & $2 \mathrm{~h}$ & 17 & $2-3$ \\
\hline \multirow{3}{*}{ Arrhytmia } & mitdb & $30 \mathrm{~m}$ & 17 & 2 \\
& svdb & $30 \mathrm{~m}$ & 17 & 2 \\
& incartdb & $30 \mathrm{~m}$ & 17 & 12 \\
\hline \multirow{2}{*}{ ST-T changes } & edb & $2 \mathrm{~h}$ & 17 & 2 \\
& ltstdb & $21-24 \mathrm{~h}$ & 17 & $2-3$ \\
\hline \multirow{2}{*}{ Stress } & thew & $15 \mathrm{~m}$ & 17 & 12 \\
& stdb & $10-40 \mathrm{~m}$ & 17 & 2 \\
\hline \multirow{3}{*}{ Long Term } & ltdb & $14-22 \mathrm{~h}$ & 7 & 2 \\
& $\mathrm{sddb}$ & $23 \mathrm{~h}$ & 12 & 2 \\
& ltafdb & 1 day & 17 & 2 \\
\hline Total & & $\sim 92$ days & 178 &
\end{tabular}

$\mathbf{p}$ immerse in a noisy signal $\mathbf{s}$. One of its limitations can be that $\mathbf{p}$ must be a priori known, which is an important drawback at the moment of designing and automatic algorithm. In this work we proposed to overcome this limitation with the use of a two step algorithm. 1) First we perform an approximation step, which consists of generating a generic p to perform an approximate detection. 2) The refinement step consists on repeating step 1), but with a set of patterns $\mathbf{P}$ estimated from the results of the first step, as described below. The scheme of Figure 1 attempt to ease the explanation.

The first part of the approximation step 1) consists in calculating the detection signal, $a(n)$, using eq. 1 , with a generic impulsive pattern calculated as

$$
\begin{aligned}
& p(n)=g(n) \cdot \frac{\partial}{\partial n} \cdot g(n), \\
& g(n)=e^{-\frac{n^{2}}{2 \sigma^{2}}}, \sigma=\frac{N-1}{5} .
\end{aligned}
$$

Note that the generic $p(n)$ is the derivative of a Gaussian pattern with a user-defined width $N$. The derivative was approximated by finite differences of the sequence $g$. The detection signal $a$ is then smoothed with an averaging lowpass FIR filter of integer length $M=1.2 * N$, obtaining $a_{s}(n)$. In the following block, all the maxima extreme values of $a_{s}$ above a threshold $t_{a}=P_{30}\left\{a_{s}\right\}$, are located and stored in

$$
\mathbf{b}=\underset{\mathbf{n}}{\operatorname{argmax}}\left\{\mathbf{a}_{\mathbf{s}}(\mathbf{n}) \geq \mathbf{t}_{\mathbf{a}}\right\} .
$$

The value of $t_{a}$ was calculated as the 30th. percentile of $a_{s}$, and was empirically adopted in order to sample the noise floor. Another restriction required for the values of $\mathbf{b}$, is that the occurrence of two subsequent maxima must be greater or equal than a user-defined parameter $m_{0}$. In case this condition is not satisfied, only the

$$
b_{i}=\max _{n \in W}\left\{a_{s}(n)\right\}
$$

with

$$
W=\left\{j=1, \cdots, B \wedge \forall k: b_{k} \geq b_{j} \wedge b_{k} \leq\left(b_{j}+m_{0}\right)\right\}
$$

are stored. Once all the $B$ relevant extreme values are stored in $\mathbf{b}$, the algorithm continues with the calculation of $t_{b}$, a second threshold used for the final impulse detection in signal $a_{s}$. The value of $t_{b}$ is calculated from $\mathbf{h}_{\mathbf{b}}$, i.e. the curve defined by the histogram values of $\mathbf{b}$. The histogram was calculated by counting occurrences over an ad-hoc equally spaced grid, with a grid step calculated as

$$
\begin{aligned}
g_{s} & =\operatorname{med}\left\{\frac{\partial}{\partial \mathrm{i}} \mathrm{p}(\mathrm{i})\right\} \\
p(i) & =P_{q_{i}}\left\{a_{S}\right\}, q=1,2 \cdots, 100 .
\end{aligned}
$$

Note that $g_{s}$ is the median finite difference of an uniformly sampled percentile grid over the values of $a_{s}$. The value of $g_{s}$ favors an adequate representation of $\mathbf{h}_{\mathbf{b}}$, in order to perform the detection of the first minimum, $t_{b}$, corresponding to the final detection threshold to use with the detection signal $a_{s}$. The approximation step concludes after storing the $M_{0}$ detections in

$$
\mathbf{d}_{0}=\left(n_{0}, n_{1}, \cdots, n_{M_{0}}\right)
$$

being $n_{i}=\underset{n}{\arg }\left\{a_{s}(n) \geq t_{b}\right\}$.

The refinement step starts by seeking $Q$ locations in $\mathbf{d}_{0}$ corresponding to representative patterns based on a ranking criterion. The criterion quantifies the stability of the 1) rhythm and 2) morphology of the pattern. The selected patterns should be those whose stability and frequency (or rhythm) are more stable. The quantification of the rhythm stability starts by calculating the instantaneous rhythm

$$
\mathbf{r}_{0}=\left(r_{0}, r_{1}, \cdots, r_{M_{0}-1}\right),
$$

using the locations in $\mathbf{d}_{0}$ being $r_{i}=n_{i+1}-n_{i}$. Then, from $\mathbf{r}_{0}$ an estimate of the instantaneous median rhythm $\tilde{\mathbf{r}}_{0}$ was calculated where $\tilde{r}_{i}=\operatorname{med}\left\{\mathbf{r}_{\mathbf{S}}\right\}$, being $\mathbf{r}_{\mathbf{S}}$ the sequence of rhythm values included in a centered window $S$ around the $i$-th value. With these two sequences, an scatter sequence $\tilde{\mathbf{s}}_{0}$ was calculated, with elements

$$
\tilde{s}_{i}=\frac{\left(r_{i}-\tilde{r}_{i}\right)}{\tilde{r}_{i}} .
$$

The value $\tilde{s}_{i}$ represents the instantaneous relative change in rhythm. Finally a two column matrix $\mathbf{T}_{\mathbf{j}}$ with the startend of each stable segment is constructed. An $\tilde{s}_{i}$ element is considered stable if its value is less than the median of $\tilde{\mathbf{s}}_{0}$. Then, an stable segment is defined as a sequence of adjacent and stable $\tilde{s}_{i}$, whose length is grater than a time $w_{s}$. The start and ending sample of all $L_{0}$ segments that meet this condition are stored in $\mathbf{T}_{\mathbf{j}}$. A first rhythm score 


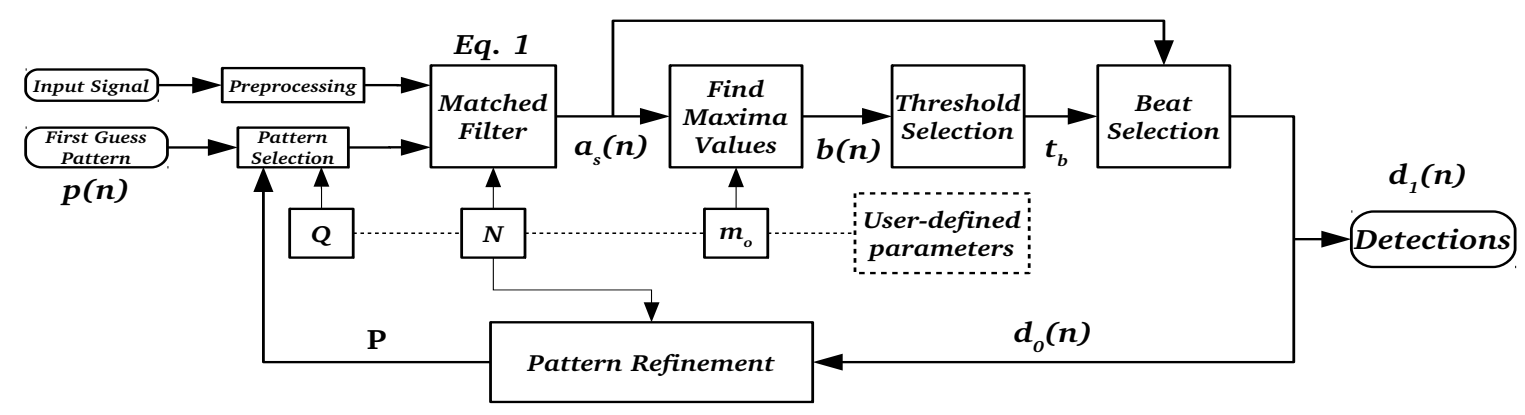

Figure 1. Block diagram of the proposed algorithm.

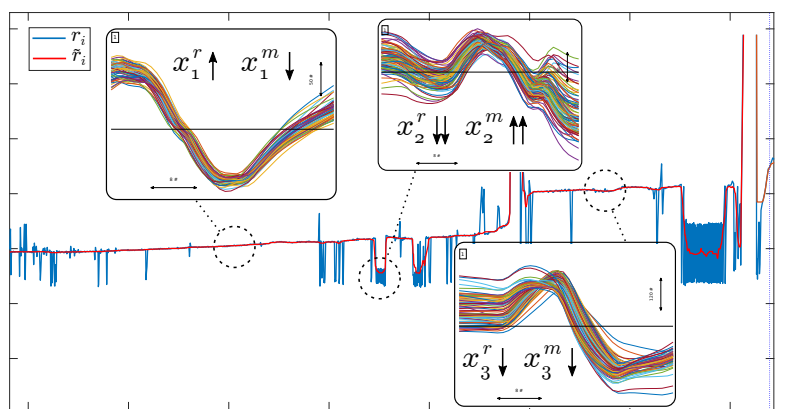

Figure 2. Rhythm evolution in a rodent recording. Qualitative example of the rhythm and morphology scores in stable (1 and 3) and unstable rythm segments. The 3rd segment presents morphology unstability.

$x_{j}^{r}$ with $j=1,2, \cdots, L_{0}$ is calculated for each row in $\mathbf{T}_{\mathbf{j}}$.

The morphology score $x_{j}^{m}$ was also calculated for each of the $L_{0}$ stable segments. For each segment, a $D_{j} \times N$ pattern matrix $\mathbf{U}_{\mathbf{j}}$ is constructed with all the $D_{j}$ detected patterns of length $N$ found. The mean variance across the ensemble of patterns was used as morphology score and was calculated as

$$
x_{j}^{m}=\underset{x}{\operatorname{Mean}}\left[\operatorname{Var}\left(\mathbf{U}_{\mathbf{j}}\right)\right],
$$

where $y$ means the column (or ensemble) direction and $x$ the row (or time) direction of matrix $\mathbf{U}_{\mathbf{j}}$. With both scores the final score was simply $x_{j}^{f}=x_{j}^{r}+x_{j}^{m}$, and was used to select the $Q$ most promising rows in $\mathbf{T}_{\mathbf{j}}$ (stable segment). A qualitative example can be observed in Figure 2. The refinement step continues by calculating a representative pattern from each segment

$$
\mathbf{P}=\left(\mathbf{p}_{\mathbf{0}}, \mathbf{p}_{\mathbf{1}}, \cdots, \mathbf{p}_{\mathbf{Q}}\right) .
$$

The Woody method was used to calculate $\mathbf{p}_{\mathbf{k}}$ patterns from the $\mathbf{d}_{0}$ detections $[2,5]$. Note that $\mathbf{p}_{\mathbf{0}}$ is the generic pattern defined in equation 2. Once the pattern matrix $\mathbf{P}$ is calculated, the algorithm concludes by repeating the approximation step for each $\mathbf{p}_{\mathbf{k}}$ and producing the corresponding detection vector $\mathbf{d}_{\mathbf{k}}$ for $k=1,2, \cdots, Q$. We only considered the detections from the first ranked pattern $\mathbf{d}_{\mathbf{1}}$ for the performance calculation presented in the next section.

The experimentation was performed with the following user defined parameters: for the human set $m_{o}=300 \mathrm{~ms}$, $N=60 \mathrm{~ms}$ and $Q=2$; for the rodent set $m_{o}=120 \mathrm{~ms}, N=20 \mathrm{~ms}$ and $Q=2$.

The evaluation of the proposed algorithm was performed with respect to the gold standard annotations included in each database. It was calculated by means of the sensitivity and positive predictive value for each recording. The median results for each set of the corpus were calculated for a global performance assessment.

\section{Results}

The results obtained for both evaluation sets are summarized in Table 2. The worst cases for each database are shown, and the median result for each set of the corpus. In this work we also evaluated the gqrs detector [1] and a threshold independent algorithm [6] under the rodent set. Results where $S=72.1 ; P=61.2$ for the gqrs, and $S=74.5$; $P=87.4$ for the threshold independent algorithm.

\section{Discussion and conclusions}

In this work we evaluated an algorithm for pseudo periodic patterns. As explained above, the pattern description was made with two time parameters: the pattern duration $(N)$ and the minimum time between successive patterns $\left(m_{0}\right)$. This representation achieved promising results for the detection of QRS complexes in human and rodent recordings. The evaluation set includes recordings from 12 human ECG databases publicly available [1,7], as shown in Table 1. Another set of 17 pseudo ECG recordings of 
Table 2. Worst result for each database in the corpus. Median values in percent.

\begin{tabular}{|c|c|c|c|}
\hline Database & Recording Name & $S$ & $P$ \\
\hline edb & $\mathrm{e} 0116$ & 99.5 & 50.6 \\
\hline fantasia & f1o04 & 99.9 & 99.7 \\
\hline INCART & I03 & 90.0 & 83.8 \\
\hline ltafdb & 45 & 88.1 & 49.6 \\
\hline ltdb & 14179 & 99.8 & 100 \\
\hline ltstdb & s30801 & 87.9 & 90.3 \\
\hline mitdb & 201 & 96.6 & 99.9 \\
\hline nsrdb & 16272 & 98.3 & 92.7 \\
\hline sddb & 49 & 99.0 & 88.3 \\
\hline $\mathrm{stdb}$ & 843 & 99.6 & 99.9 \\
\hline svdb & 854 & 100 & 99.3 \\
\hline thew & $*$ & 99.4 & 99.5 \\
\hline \multicolumn{2}{|c|}{ Human set } & 100 & 99.9 \\
\hline rodendb & \#6 & 77.0 & 57.2 \\
\hline rodendb & \#16 & 99.2 & 42.2 \\
\hline \multicolumn{2}{|c|}{ Roden set } & 97.1 & 79.1 \\
\hline
\end{tabular}

*25_PR01_121003_4

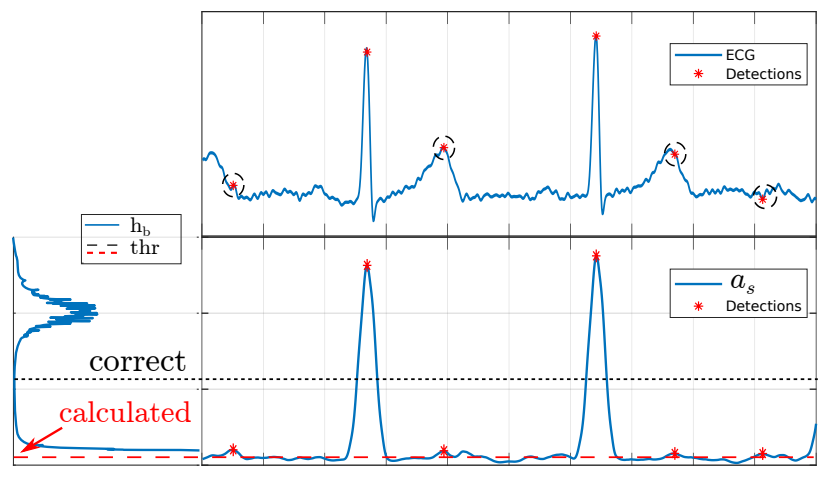

Figure 3. Case of failure: Bad threshold calculation. Example taken from recording 0116 from edb database.

explanted rodent hearts [4] was used to evaluate the generality of the pattern description.

The results presented in Table 2 show that the algorithm achieved a very good overall performance over a wide set of recordings, obtaining a $S=100$ and $P=99.9$ in the whole human set. In the same table, the worst cases were selected in order to visualize where the algorithm requires further improvement, despite the fact that the median performances are very promising. We observed in those cases that the algorithm failed calculating the threshold $t_{b}$, as is shown in Figure 3, and where a proper threshold should be located. During real-life use of the algorithm, these kind of errors are easy-to-correct, since the value of $t_{b}$ could be corrected by expert assistance, if available.

The results in the rodent set were not as good as in the human set, in Table 2 the median performance show an slight decrease in $S=97.1$ and a more important decrease in $P=79.1$. This performance drop could be explained in first place by the severe changes in morphology and rhythm observed in rodent recordings, as shown in Figure 2. Another explanation can be the experimental setup, i.e. explanted hearts subject to severe changes in the potassium concentration to induce ventricular fibrillation. It is certainly a difficult scenario for any automatic algorithm, as said above when comparing the performances with other public algorithms, where the proposed algorithm is the best performing.

The implementation of the proposed algorithm, with more detailed results can be found in [8] for comparison. Despite the limitations described, the algorithm achieved promising results with only two physiologicallymeaningful parameters, in a broad set of ECG recordings of very different nature, with the additional capability of further adaptation provided by expert assistance.

\section{Acknowledgments}

This work was supported by projects ASUTNBA0003860 and ICUTNBA0004864 from UTN FRBA.

\section{References}

[1] Goldberger AL, et al. PhysioBank, PhysioToolkit, and PhysioNet: Components of a new research resource for complex physiologic signals. Circulation 2000;101(23):e215-e220.

[2] Sörnmo L, Laguna P. Bioelectrical Signal Processing in Cardiac and Neurological Applications. Elsevier, 2005. ISBN 0-12-437552-9.

[3] Elgendi M, et al. Revisiting qrs detection methodologies for portable, wearable, battery-operated, and wireless ecg systems. PLOS ONE 01 2014;9(1):1-18.

[4] Diez ER, et al. Melatonin, given at the time of reperfusion, prevents ventricular arrhythmias in isolated hearts from fructose fed rats and spontaneously hypertensive rats. Journal of Pineal Research ;55(2):166-173.

[5] Harris EK, Woody CD. Use of an adaptive filter to characterize signal-noise relationships. Computers and Biomedical Research 1969;2(3):242-273.

[6] Ramakrishnan AG, Prathosh AP, Ananthapadmanabha TV. Threshold-independent qrs detection using the dynamic plosion index. IEEE Signal Processing Letters May 2014; 21(5):554-558. ISSN 1070-9908.

[7] Couderc JP. The telemetric and holter ECG warehouse initiative THEW. URL thew-project.org.

[8] Implementation of the proposed algorithm. URL https: //github.com/ajs93/aip.git.

Address for correspondence:

Augusto Santini, asantini@ est.frba.utn.edu.ar Medrano 951 (C1179AAQ) C.A.B.A., Argentina 